\title{
Development of a Sexually Dimorphic Projection from the Bed Nuclei of the Stria Terminalis to the Anteroventral Periventricular Nucleus in the Rat
}

\author{
Leslie A. Hutton, ${ }^{1}$ Guibao Gu, ${ }^{1}$ and Richard B. Simerly ${ }^{1,2}$ \\ ${ }^{1}$ Division of Neuroscience, Oregon Regional Primate Research Center, Beaverton, Oregon 97006, and 2Program in \\ Neuroscience, Oregon Health Sciences University, Portland, Oregon 97201
}

The principal nucleus of the bed nuclei of the stria terminalis (BSTp) is larger in male rats and conveys olfactory information relevant for reproduction to the hypothalamus. In males, the BSTp provides a massive projection to the anteroventral periventricular nucleus of the preoptic region (AVPV), which in contrast to most sexually dimorphic nuclei contains more neurons in female rats. Injections of the anterograde tracer Phaseolus vulgaris leucoagglutinin into the BSTp of adult female rats failed to demonstrate the strong projection to the AVPV observed previously in males. The ontogeny of this robust sex difference was examined by using the axonal marker Dil. The projection from the BSTp to the AVPV is established between postnatal day 9 (P9) and P10 in male rats and seems to be maintained during the juvenile period. Although labeled fibers extended from the BSTp toward the preoptic region in both male and female neonates, a similar connection with the AVPV was not apparent in female rats at any of the ages studied, and the density of labeled axons in the AVPV of P10 males was 20 -fold greater than that of P10 females. A projection from the BSTp to the medial preoptic nucleus was also weaker in females but was much more substantial than that to the AVPV. These findings suggest that a sex- and region-specific activity influences the development of the projection from the BSTp to the AVPV, producing a sexually dimorphic architecture in pathways that convey olfactory information to the hypothalamus.

Key words: sexual differentiation; preoptic region; Dil; connections; dimorphic; development; bed nucleus of the stria terminalis
Sex steroid hormones exert profound effects on mammalian forebrain development, including regulation of the volume of sexually dimorphic nuclei, the density of certain classes of synapses, and the number of neurotransmitter-specific neurons (for review, see Simerly, 1990; Matsumoto, 1991; Segovia and Guillamón, 1993). In addition to regulating the number of neurons in sexually dimorphic nuclei, sex steroids seem to regulate the development of sexually dimorphic patterns of connectivity in forebrain regions thought to play important roles in mediating reproductive function. Raisman and Field (1971) were the first to demonstrate a sex difference in the density and organization of synapses in the preoptic region of the rat; sexually dimorphic patterns of connectivity in the medial nucleus of the amygdala and in the ventrolateral part of the ventromedial hypothalamic nucleus have also been identified (Matsumoto, 1991). However, a similar sex difference in synaptic connections was not found in the dorsomedial part of the ventromedial nucleus (Matsumoto and Arai, 1986), indicating that the development of sexually dimorphic connections is highly localized and region specific.

In rodents, pheromonal olfactory information is conveyed from the vomeronasal organ to the accessory olfactory bulb and relayed then to the hypothalamus along a sexually dimorphic path-

Received Sept. 11, 1997; revised Jan. 30, 1998; accepted Feb. 4, 1998.

This work was supported by National Institutes of Health Grants MH49236, RR00163, and HD18185. We thank Meigan Crabtree for expert technical assistance and C. Houser for preparation of this manuscript. We are also grateful to Dr. S. Amara of the Vollum Institute (Oregon Health Sciences University) for use of the Bio-Rad MRC 1000 confocal microscope.

Correspondence should be addressed to Dr. Richard B. Simerly, Program in Neuroscience, Oregon Health Sciences University, 3181 Southwest Sam Jackson Park Road, Portland, OR 97201.

Copyright (C) 1998 Society for Neuroscience $\quad 0270-6474 / 98 / 183003-11 \$ 05.00 / 0$ way comprised of neuronal cell groups that are larger in male rats. Each of these regions seems to influence the expression of reproductive behavior and participate in the neural control of gonadotropin secretion (for review, see Simerly, 1990; Segovia and Guillamón, 1993). The principal nucleus of the bed nuclei of the stria terminalis (BSTp) (for parcellation and nomenclature, see Ju and Swanson, 1989) is a key component of this olfactory pathway and seems to develop under the influence of perinatal sex steroid hormones (del Abril et al., 1987). In adult animals, the BSTp contains high densities of neurons that express high levels of receptors for estrogen (ER) and androgen (AR) (Simerly et al., 1990). Moreover, the BSTp displays high levels of steroid binding, and the majority of its neurons express ER and AR mRNA during the postnatal period (Don Carlos and Handa, 1994; McAbee and Don Carlos, 1996) (R. B. Simerly, unpublished observations). Expression of androgen and estrogen receptors in the BSTp and the AVPV seems to be sexually dimorphic in rats. AR expression in the BSTp tends to be higher in males, whereas levels of ER expression are generally higher in females (Simerly et al., 1990). Because the BSTp contains high levels of the enzyme aromatase (Shinoda et al., 1994), which converts testosterone to estradiol, either receptor may mediate the effects of elevated levels of circulating testosterone on the development of BSTp neurons.

The BSTp sends strong projections to hypothalamic nuclei, such as the medial preoptic nucleus and the ventromedial hypothalamic nucleus, known to play important roles in the regulation of reproductive behavior (Simerly, 1990). In addition, the BSTp sends strong inputs to cell groups in the periventricular zone of the hypothalamus that are thought to regulate hormone secretion from the anterior pituitary (Gu and Simerly, 1997a). The antero- 
ventral periventricular nucleus of the preoptic region (AVPV) is of particular interest because it seems to represent a nodal point in the neural circuits that regulate gonadotropin secretion (Simerly, 1998). Lesions of the AVPV block spontaneous ovulation (Wiegand and Terasawa, 1982), and neurons in the AVPV seem to provide direct inputs to gonadotropin-releasing hormone (GnRH)-containing neurons in the preoptic region and to tuberoinf undibular dopaminergic neurons in the arcuate nucleus (Gu and Simerly, 1997b) that regulate the secretion of LH and prolactin, respectively (Fink, 1988; Neill and Nagy, 1994). Like the BSTp, the AVPV is sexually dimorphic and contains high densities of neurons that express estrogen receptors during the perinatal period (Don Carlos and Handa, 1994) (Simerly, unpublished observations). In contrast to the BSTp, which is larger in males, the AVPV appears to contain more neurons in female rats (Sumida et al., 1993). Thus, the connection between the BSTp and the AVPV represents a hormone-sensitive interface between the telencephalon and diencephalon. Moreover, this connection links two sexually dimorphic nuclei that have divergent developmental histories, a hormonally induced reduction of neurons in the AVPV of males concomitant with an increase in the number of neurons in the BSTp (del Abril et al., 1987; Sumida et al., 1993).

The purpose of the present study was to compare the strength of the projection from the BSTp with the AVPV in male and female rats and to study the development of this pathway. Anterograde transport studies identified a robust sexually dimorphic projection in adult animals. In male rats, the BSTp provides a massive projection to the AVPV, but in females, the AVPV seems to receive only a weak input from the BSTp. DiI axonal transport was used to define the temporal organization of the development of this pathway and to determine whether this connection is established early in both sexes and retracts in females or never achieves in females the density of innervation that is characteristic of male rats. Our results indicate that, in males, axons from the BSTp reach the AVPV on postnatal day 9 (P9) and form a dense plexus of fibers in the AVPV by P10. Although a few projection fibers from the BSTp reach the AV PV by P10 in female rats, the dense innervation pattern seen in males was not observed at any age.

\section{MATERIALS AND METHODS}

Phaseolus vulgaris leucoagglutinin transport studies in adult animals. The protocols used for the adult and neonatal studies were approved by our Institutional Committee for the Care and Use of Animals in Research and Education, in accordance with the guidelines of the National Institutes of Health and United States Department of Agriculture. The projection from the BSTp to the AVPV was traced in adult rats by placing iontophoretic injections of $P$. vulgaris leucoagglutinin (PHAL) into the BSTp and by immunohistochemically labeling axons containing the anterogradely transported lectin according to methods described elsewhere (Gu and Simerly, 1997b). Ten adult male and 10 adult female Sprague Dawley rats $(\sim 260-310 \mathrm{gm})$ received injections of PHAL in the BSTp. Briefly, a single iontophoretic injection of a $2.5 \%$ solution of PHAL in $0.1 \mathrm{M}$ sodium phosphate buffer, $\mathrm{pH} 7.4$, was made through a stereotaxically positioned glass micropipette by applying a $+4.5 \mu \mathrm{A}$ current, pulsed at $7 \mathrm{sec}$ intervals, provided by a constant current source (model CS4; Transkinetics, Canton, MA) for a period of 15-20 min. After a survival time of $14 \mathrm{~d}$ after the injection, each animal was deeply anesthetized with tribromoethanol and perfused transcardially with a solution of $4 \%$ paraformaldehyde in $0.1 \mathrm{~m}$ sodium borate buffer, $\mathrm{pH} 9.5$. The brains were immediately removed and post-fixed in the same fixative containing $10 \%$ sucrose. Frozen sections ( $30 \mu \mathrm{m}$ thick) were cut on a sliding microtome in the frontal plane and collected through the preoptic region (one section from every $120 \mu \mathrm{m}$ ). Additional sections were collected through the rostral forebrain containing the BSTp and were "quick-stained" for confirmation of the injection site location (Simerly and Swanson, 1988). Sections were incubated for $1 \mathrm{hr}$ in a 1:500 dilution of rabbit anti-PHAL that was localized with an affinity-purified goat anti-rabbit $\operatorname{IgG}$ conjugated with fluorescein isothiocyanate (FITC) (Tago, Burlingame, CA). One complete series of sections was processed for immunohistochemistry with a rabbit antiserum directed against PHAL (Dako, Carpenteria, CA) at a dilution of 1:2000. The primary antiserum was localized by using a variation of the avidin-biotin complex system (ABC) (Hsu and Raine, 1981; Hsu et al., 1981) with a commercially available kit (ABC Elite Kit; Vector Laboratories, Burlingame, CA). The sections were mounted onto gelatin-coated slides and treated with osmium tetroxide to enhance visibility of the reaction product according to methods described in detail elsewhere (Simerly and Swanson, 1988). Slides were then dehydrated, coverslipped with DPX mountant (Electron Microscopy Sciences, Fort Washington, PA), and examined with both conventional and dark-field-transmitted light microscopy. An adjacent series of sections was stained with thionin to serve as a reference series for cytoarchitectonic purposes. Only cases with injections centered within the morphological borders of the BSTp were included in the analysis. In such cases, nearly all of the PHAL-labeled neurons were contained in the BSTp, and the injections were of comparable size in the male and female animals.

DiI implants in postnatal rats. Neonatal male and female Sprague Dawley rats were anesthetized with tribromoethanol and perfused on $\mathrm{P} 0$, P2, P4, P6, P7, P9, P10, and P22 with a $4 \%$ paraformaldehyde solution, $\mathrm{pH}$ 9.5. The brains were immediately removed and stored in fixative at $4^{\circ} \mathrm{C}$ until further processing. Each brain was blocked and embedded in $1.5 \%$ agarose and sectioned from caudal to rostral to expose the BSTp without disturbing the preoptic region. Each brain block was stained with methylene blue to visualize morphological features of the exposed surface of the block, which allowed unambiguous identification of the morphological borders of the BSTp. An insect pin was used to place a small crystal of DiI $\left(1,1^{\prime}\right.$-dioctadecyl-3,3,3',3'tetramethylindocarbocyanine perchlorate; Molecular Probes, Eugene, OR) into the BSTp of each brain under visual guidance. For all implants, we selected DiI crystals that were similar in size as judged by eye when viewed under a dissecting microscope. The brain blocks were stored in $0.4 \%$ formaldehyde, and the DiI was allowed to diff use for 1 month in the dark at $37^{\circ} \mathrm{C}$. The brains were then re-embedded in $1.5 \%$ agarose, and $100-\mu \mathrm{m}$-thick sections through the preoptic area were cut on a vibratome, mounted onto poly-L-lysine-coated glass slides, and coverslipped with $10 \%$ buffered glycerol. Sections through the preoptic region were evaluated with both conventional fluorescence and confocal microscopy.

Evaluation. DiI-labeled material was viewed with a Leitz diaplan epifluorescence microscope equipped with a rhodamine filter set for viewing the orange-red DiI fluorescence. A series of $20-\mu \mathrm{m}$-thick sections through the preoptic area of a P10 male rat was used as a standard reference Nissl series. Sections of this Nissl series were used to prepare projection drawings through representative levels of the preoptic area, and the distribution of labeled fibers in experimental cases was charted onto this series of drawings for illustrative purposes. The nomenclature and subdivisions of the BST are based on that of Ju and Swanson (1989) and of Swanson (1992). In transposing our results to the standard series, we used constant observation of the experimental material to plot the distribution of fibers onto the standardized drawings to achieve the most accurate representation of the fiber distribution. Because the tissue was not Nissl stained, we used our best estimation of the location of fibers in the sections, and we must stress that the maps represent the fiber distribution derived from one to two sections projected onto a standardized drawing for each level. The drawings are not meant to represent a quantitative evaluation of the development of the projection but rather serve to illustrate the overall distribution of fibers during development.

Quantitative analysis. Confocal images of DiI-labeled fibers were collected through the AVPV and the medial part of the medial preoptic nucleus (MPNm) of P10 male and female rats by using a Bio-Rad MRC 1000 confocal microscope. For quantification, a series of 10 adjacent optical sections were collected through the AVPV of male and female rats along the rostrocaudle axis by using a $40 \times$ objective [numerical aperture (NA), 1.3; working distance, $0.8-1.3 \mathrm{~mm}$ ] and a $1 \mu \mathrm{m} \mathrm{Z}$ interval. Image analysis was performed by using Metamorph image analysis software (Universal Imaging Corporation, West Chester, PA). Maximum intensity projections of each series of confocal images were prepared and binarized according to user-defined threshold parameters that optimized detection of labeled fibers. The total number of pixels in each binarized 

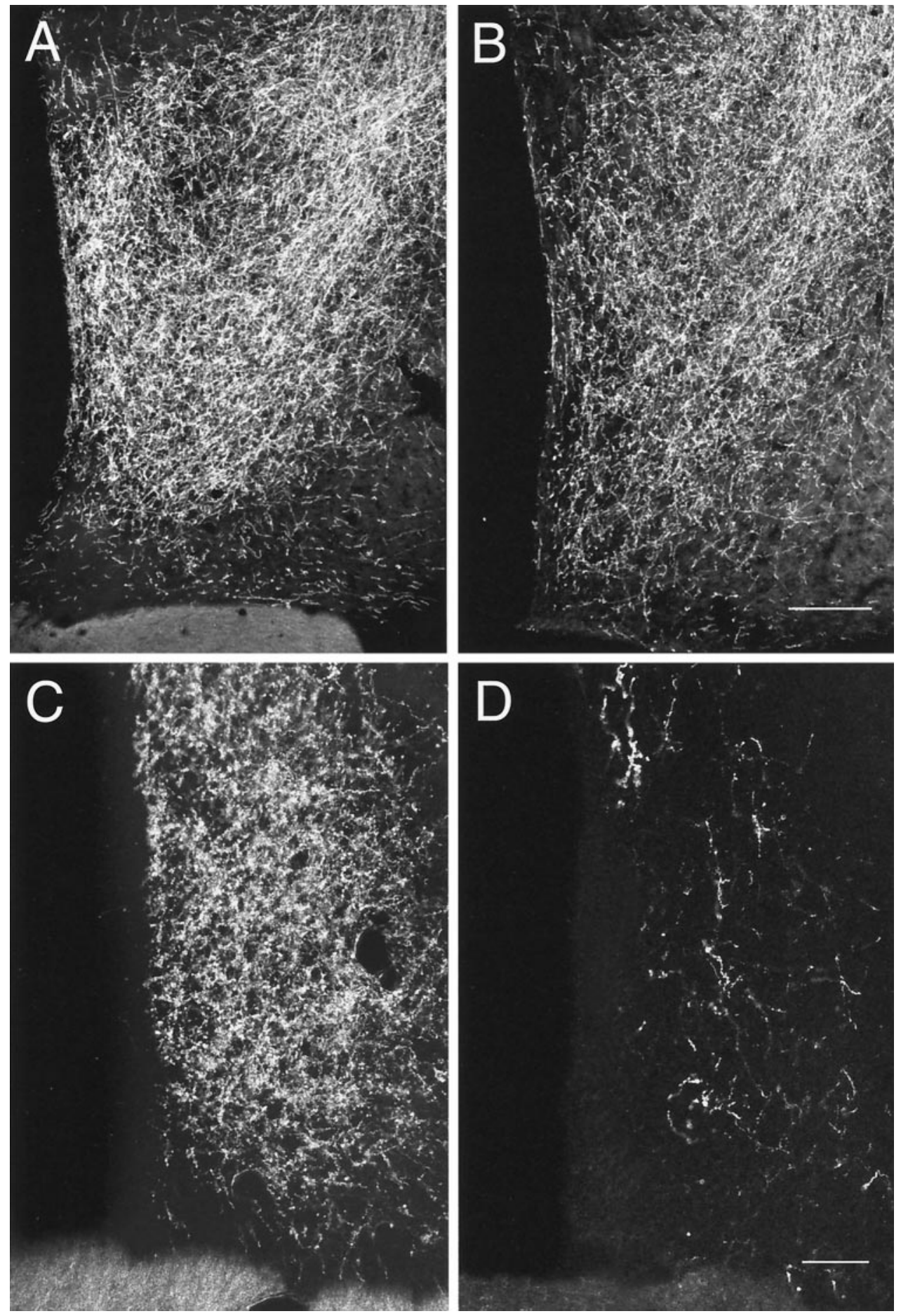

Figure 1. Dark-field photomicrographs of frontal sections through the preoptic region that compare the distribution and density of PHAL-labeled fibers in the AVPV $(C, D)$ and MPNm $(A, B)$ of adult male $(A, C)$ and female $(B, D)$ rats. Scale bars: $A, B, 20 \mu \mathrm{m} ; C, D, 50 \mu \mathrm{m}$. image was counted and used as an index of the overall density of labeled fibers in the AVPV.

\section{RESULTS}

The projection from the BSTp to the AVPV in adult male and female rats was examined using PHAL anterograde tracing. In adult male rats, injections of PHAL that were centered in the BSTp labeled fibers that either pass ventrolaterally toward the medial forebrain bundle and through the substantia innominata or pass ventromedially to innervate structures in the preoptic region. The latter fibers form a particularly dense plexus of labeled axons and fiber terminals in the MPNm and AVPV (Fig.
$1 A, C$, respectively). Similar injections of PHAL into the BSTp of adult female rats also labeled a plexus of fibers in the MPNm that appeared to be somewhat less dense than was that of males (Fig. $1 A, B)$. However, the AVPV of females contained only a few labeled fibers in marked contrast to the high density of labeled fibers and terminals found in the AVPV of males (Fig. $1 C, D$ ). Evaluation of the PHAL injection sites revealed that the placement was similar when male and female cases were compared (Fig. 2).

To examine the development of the sexually dimorphic projection from the BSTp to the AVPV, we used DiI axonal labeling. 

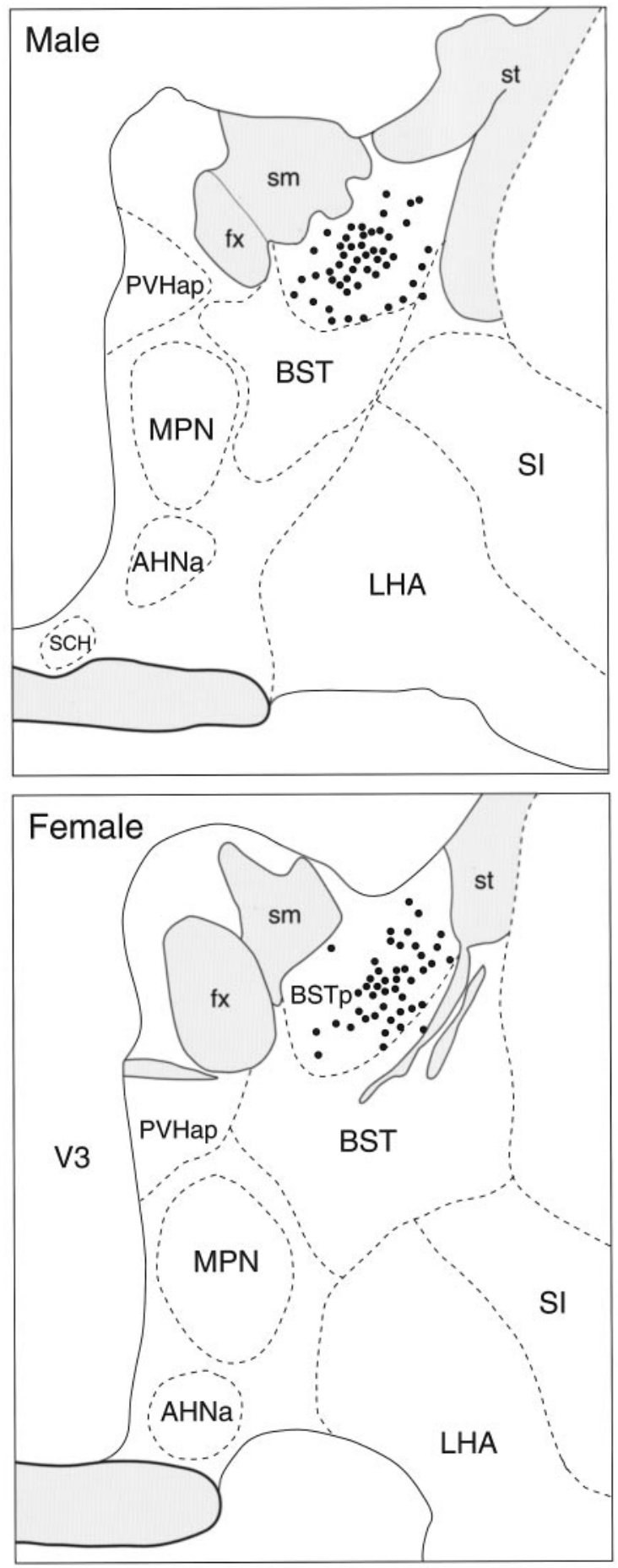

Figure 2. Camera lucida drawings that show the distribution of labeled neurons ( filled circles) after injection of $P$. vulgaris leucoagglutinin in the $B S T p$ of an adult male (top) and female (bottom) rat. AHNa, Anterior hypothalamic nucleus, anterior part; $B S T$, bed nuclei of the stria terminalis; $B S T p$, principal nucleus of the BST; $f x$, fornix; $L H A$, lateral hypothalamic area; MPN, medial preoptic nucleus; PVHap, paraventricular nucleus of the hypothalamus, anterior parvicellular part; SI, substantia innominata; $s m$, stria medullaris; $s t$, stria terminalis; $V 3$, third ventricle; $\mathrm{SCH}$, superchiasmatic nucleus; shaded grey areas, fibertracts; dashed lines, borders of nuclei.
Brains with DiI crystals centered in the BSTp were obtained from $\mathrm{P} 2(n=3), \mathrm{P} 4(n=3), \mathrm{P} 7(n=6), \mathrm{P} 9(n=3)$, and P10 $(n=4)$ male rats. In these cases, the crystalline deposits of DiI were confined to the BSTp and were $\sim 25-35 \mu \mathrm{m}$ in diameter. Slight differences in crystal size probably do not account for the differences we observed between postnatal ages and sexes because even a small DiI crystal placed in the tissue probably saturates the area adjacent to the implant with dye. When the DiI crystal was centered outside the morphological borders of the BSTp, a distinctly different pattern of labeling was observed with very few fibers entering the medial preoptic area, suggesting that diffusion of DiI beyond the morphological borders of the BSTp did not contribute to the labeling observed in the AVPV or MPNm. When the DiI crystal was centered in the BSTp of P4-P10 rats, labeled fibers projecting from the BSTp traveled along two main routes, a ventrolateral pathway that extended from the BSTp toward the medial forebrain bundle and substantia innominata and a ventromedial pathway that entered the medial preoptic area (Figs. 3, 4). Comparable implants resulted in bundles of labeled fibers in both the medial and lateral pathways, as well as labeled fibers in the MPNm. If the DiI crystals were centered outside the borders of the BSTp, the medial and lateral pathways and the terminal field in the MPNm were not labeled. It is unlikely that variability in the placement of the DiI crystal could account for the observed differences at each age, because our results were consistent within each group.

Labeled fibers projecting from the BSTp into the medial preoptic area were sparse in P2 and P4 males. In P4 animals, the ventrolateral and ventromedial pathways were evident, and labeled fibers extended beyond the borders of the BST into the preoptic area but did not reach either the MPNm or the AVPV (Figs. 3, 4, P4). In contrast to the labeling pattern observed in P4 males, labeled fibers did not extend beyond the morphological border of the BST and did not enter the medial preoptic area in P2 males. In P7 males, labeled fibers from the BSTp extended well into the medial preoptic area and were distributed along the lateral border of the MPN but were sparsely distributed within the MPNm (Figs. 3, 4, P7). The projections to the AVPV and MPNm develop rapidly between P9 and P10. The pattern of labeled fibers in the medial preoptic area of $\mathrm{P} 9$ males resembled that seen in P10 males (see below). Although both the MPNm and AVPV contained labeled fibers in P9 males, the density was much less than that observed in these nuclei in P10 males, and the distribution was much less uniform (Figs. 3, 4, P9). We did observe labeled fibers projecting through the fornix in cases in which the DiI crystal was placed immediately adjacent to the fornix but still within the confines of the BSTp. We have represented this pattern of labeled fibers in our illustrations; however, these fibers do not appear to originate in the BSTp because they were not present in all cases.

In male rats perfused on $\mathrm{P} 10$, the pattern of projections from the BSTp to the preoptic region resembled that of adult males with a dense plexus of labeled fibers found in both the MPNm and AVPV (Figs. 3, 4, P10). The density of labeled fibers in the MPNm was much greater than was that in the MPN1 (Fig. 4, P10). Similarly, a higher density of labeled fibers was observed in the AVPV than in adjacent parts of the preoptic periventricular nucleus. The same mature pattern of projections from the BSTp was also observed in P22 males. Implants placed rostral, caudal, or ventral to the BSTp produced distinctly different labeling patterns compared with that resulting from implants centered in 

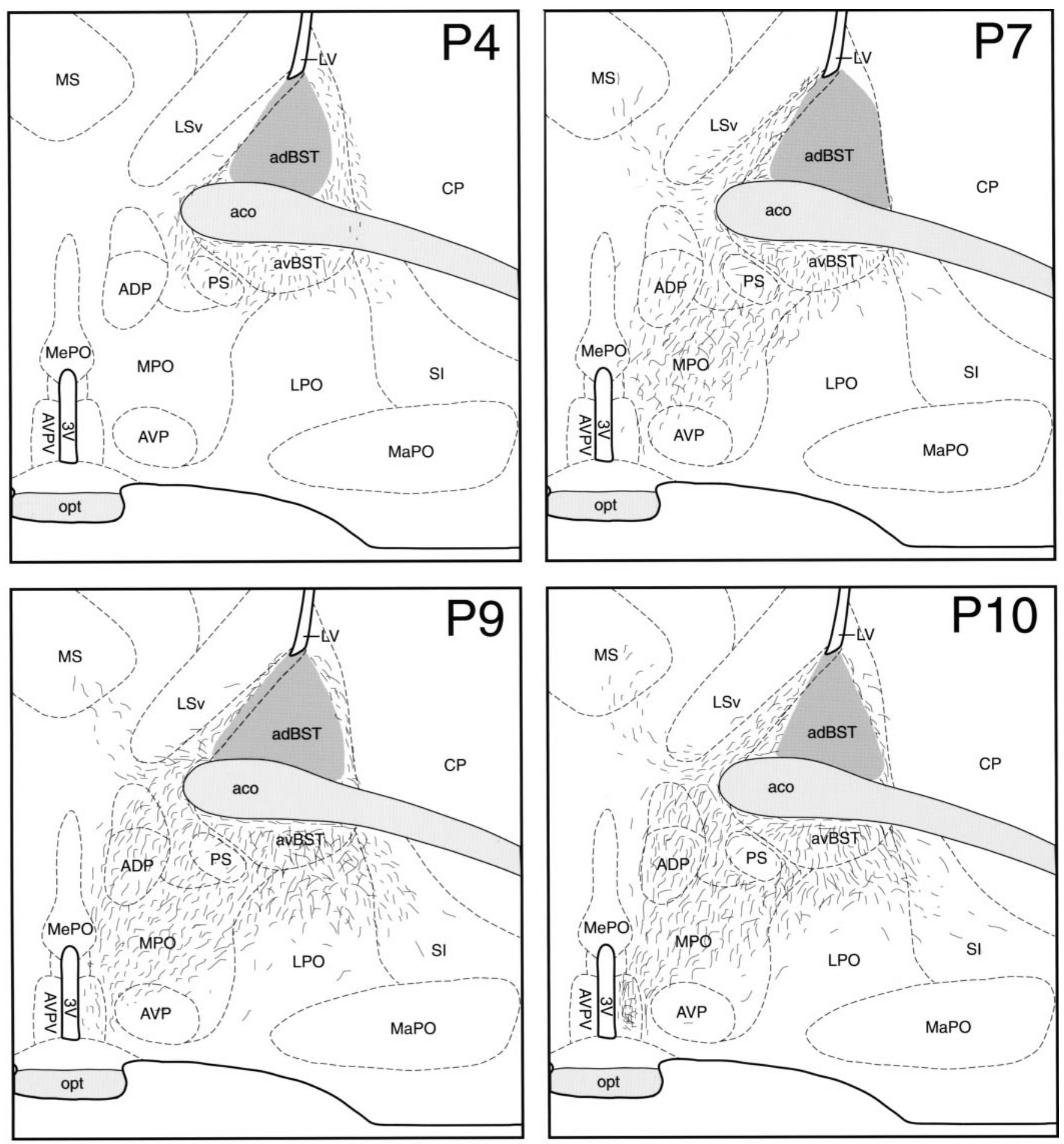

Figure 3. Preoptic projections of the BSTp. AVPV, The distribution of DiI-labeled fibers (short lines) at the level of the AVPV in male rats perfused on postnatal days $4(P 4), 7(P 7), 9(P 9)$, and $10(P 10)$ was plotted onto a standard series of drawings derived from thionin-stained sections through the preoptic region of a 10-d-old male rat. Retrogradely labeled neurons have been omitted for clarity. The dark gray region in the BST represents the area of DiI diff usion from the implant site. $3 V$, Third ventricle; $a c o$, anterior commissure; adBST, anterodorsal nucleus of the BST; $A D P$, anterodorsal preoptic nucleus; $a v B S T$, anteroventral nucleus of the BST; $A V P$, anteroventral preoptic nucleus; $A V P V$, anteroventral periventricular nucleus; $C P$, caudoputamen; $L P O$, lateral preoptic area; $L S v$, lateral septal nucleus, ventral part; $L V$, lateral ventricle; $M a P O$, magnocellular preoptic nucleus; $M e P O$, median preoptic nucleus; $M P O$, medial preoptic area; $M S$, medial septum; opt, optic tract; $P S$, parastrial nucleus. See Figure 2 for additional abbreviations.

the BSTp. Implants centered lateral to the BSTp did not label fibers in the medial preoptic area.

Similar DiI experiments performed in female rats perfused on P10 demonstrated the development of a sexual dimorphism in the projection from the BSTp to the AVPV (Fig. 5). Implant sites centered in the BSTp of three P10 females were obtained that were comparable with those obtained in P10 males (Fig. 6). Crystalline deposits of DiI centered within the morphological 

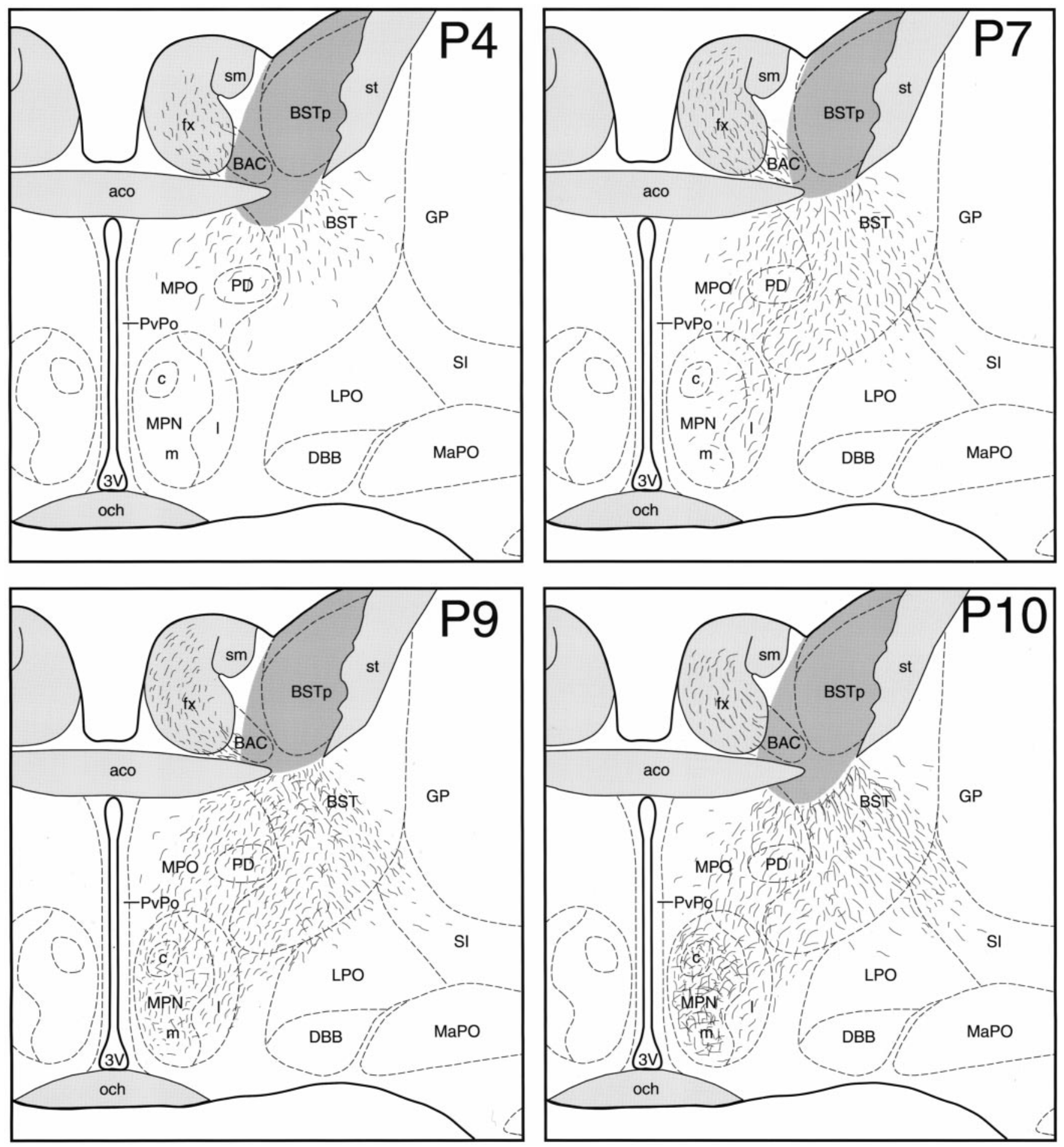

Figure 4. Preoptic projections of the BSTp. MPN, Illustrations show the distribution of DiI-labeled fibers at the level of the MPN in male rats perfused on $P 4, P 7, P 9$, and $P 10$. The dark gray area in the BST represents the area of DiI diff usion immediately adjacent to the implant site. $B A C$, Bed nucleus of the anterior commissure; $D B B$, nucleus of the diagonal band; $G P$, globus pallidus; $M P N c$, medial preoptic nucleus, central part; $M P N l$, medial preoptic nucleus, lateral part; $M P N m$, medial preoptic nucleus, medial part; och, optic chiasm; $P D$, posterodorsal preoptic nucleus; $P v P o$, preoptic periventricular nucleus. See Figures 2 and 3 for additional abbreviations.

borders of the BSTp labeled fibers that passed along the ventrolateral and ventromedial pathways. However, in contrast to our findings in P10 males, the AVPV did not contain many labeled fibers in females, although fibers were observed along the dorsal and lateral borders of the AVPV (Figs. 5, 7C,D). The density of labeled fibers in the MPNm of P10 females was also less than that observed in the P10 male cases (Fig. 8), with the greatest density of labeled fibers along its dorsal and lateral borders (Fig. 8). A similar pattern of labeled fibers projecting from the BSTp was observed in P22 females. Analysis of confocal images revealed a 20-fold difference in labeled fiber density in the AVPV between P10 male and female rats (Fig. 9). Implants of DiI centered in the 

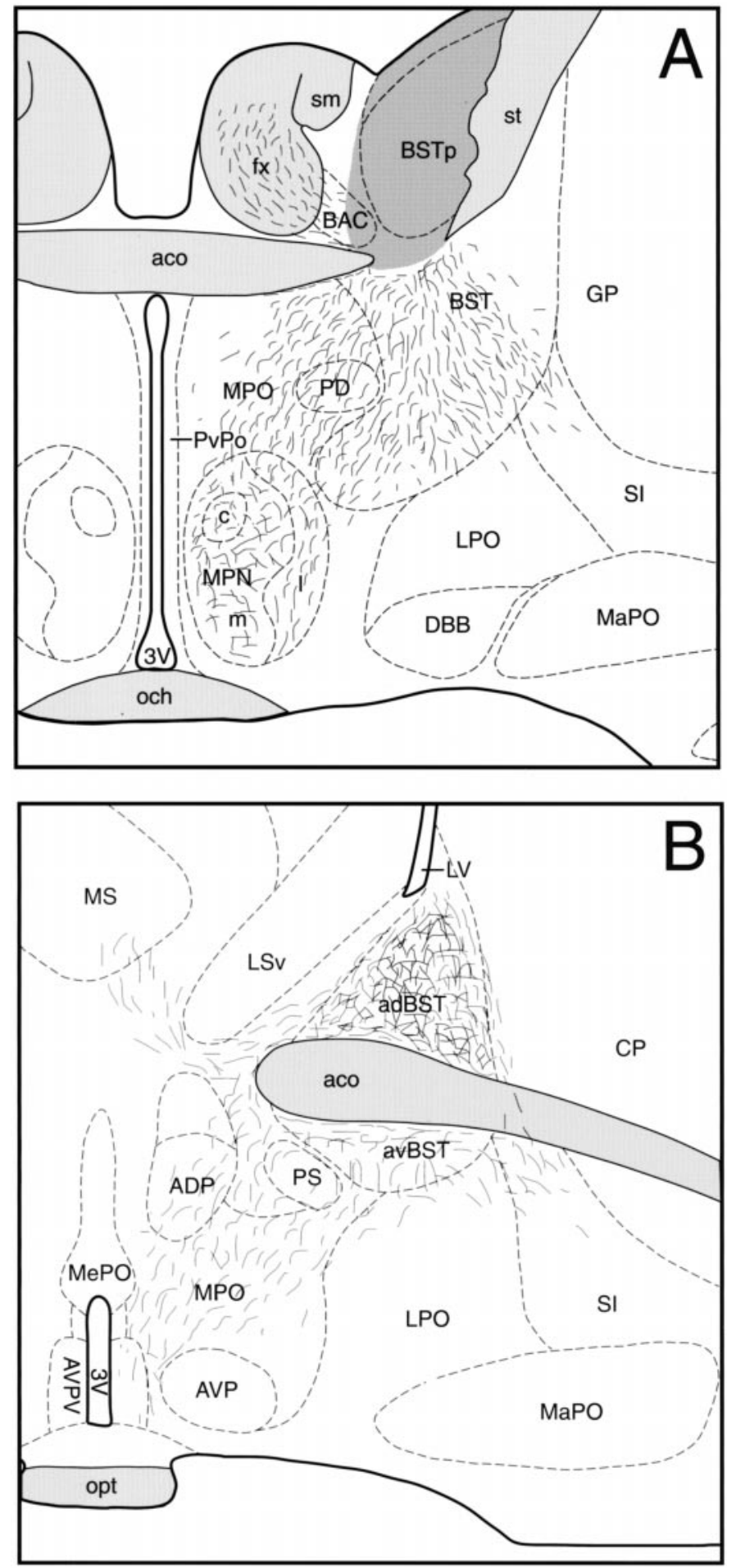

Figure 5. Sexually dimorphic projections of the BSTp. Illustrations show the density and distribution of DiI-labeled fibers projecting from the BSTp to the AVPV $(A)$ and MPNm $(B)$ in female rats perfused on P10. The dark gray region represents the area of DiI diffusion from the implant site. See Figures 2-4 for abbreviations.

BSTp of female rats perfused on P2 $(n=3), \mathrm{P} 4(n=1)$, or P6 $(n=3)$ also failed to label projections from the BSTp into the AVPV or MPNm. In both male and female cases, the MPNm contained labeled fibers only when the DiI crystal was placed in the BSTp. This observation supports our conclusion that the
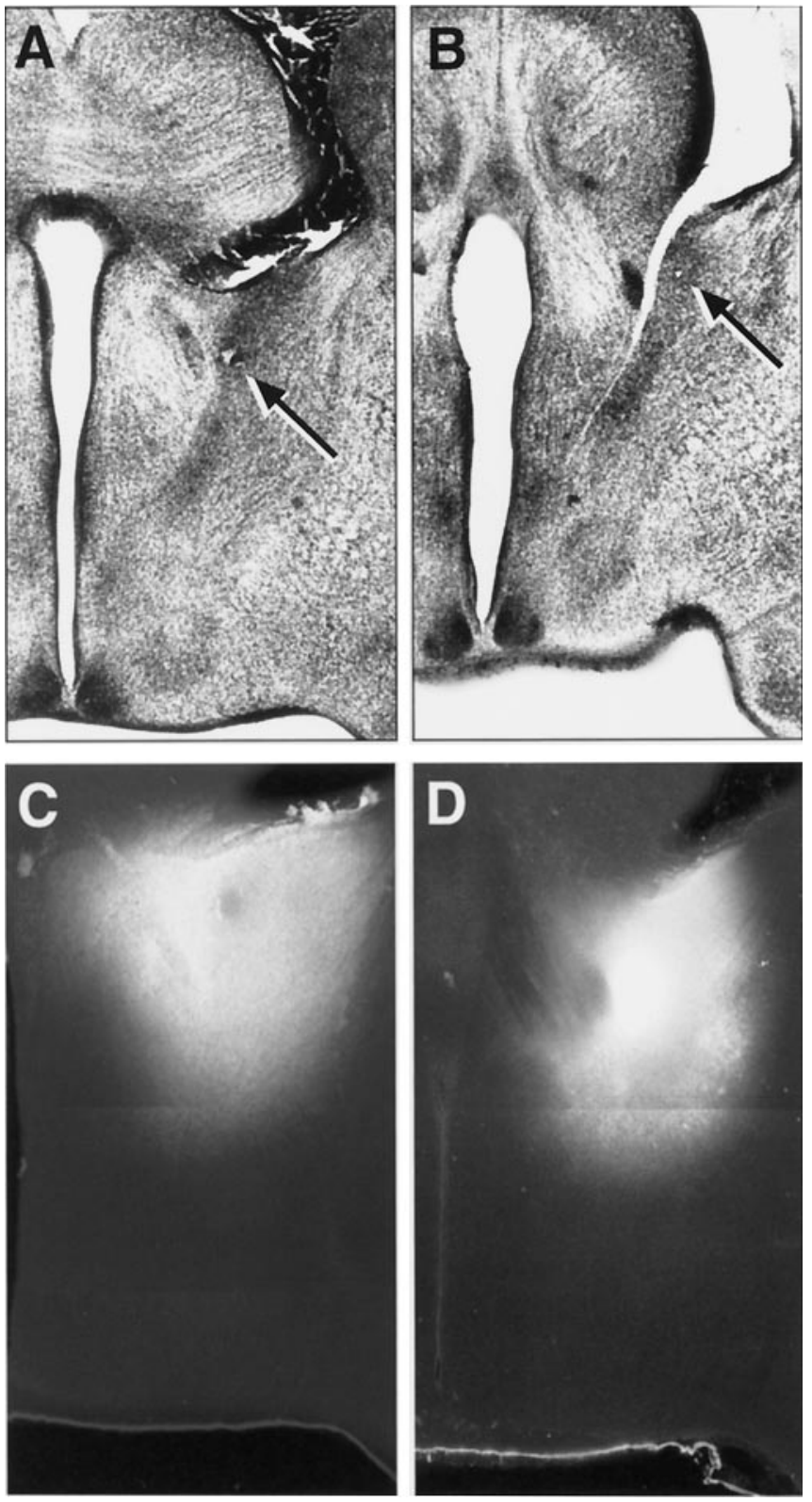

Figure 6. $A, B$, Low magnification images of Nissl-stained sections showing the precise placement of the DiI crystal in the BSTp of a P10 male $(A)$ and a P10 female $(B)$ rat. The arrows indicate the location of the center of the DiI implant. $C, D$, Low magnification, combined dark-field and fluorescence image montages showing the appearance and distribution of DiI in comparable implants obtained in P10 male $(C)$ and female $(D)$ rats.

BSTp implants were comparable in male and female rats. Moreover, the consistency of the results obtained in P10 males and P10 females suggests that it is unlikely that the observed differences are attributable to differences in the placement of the DiI crystals.

\section{DISCUSSION}

The projection from the BSTp to the AVPV represents one of the most robust morphological sex differences identified to date. The demonstration of morphological or neurochemical sexual dimorphisms in connectivity has usually depended on rigorous quantification, often at the ultrastructural level (Raisman and Field, 

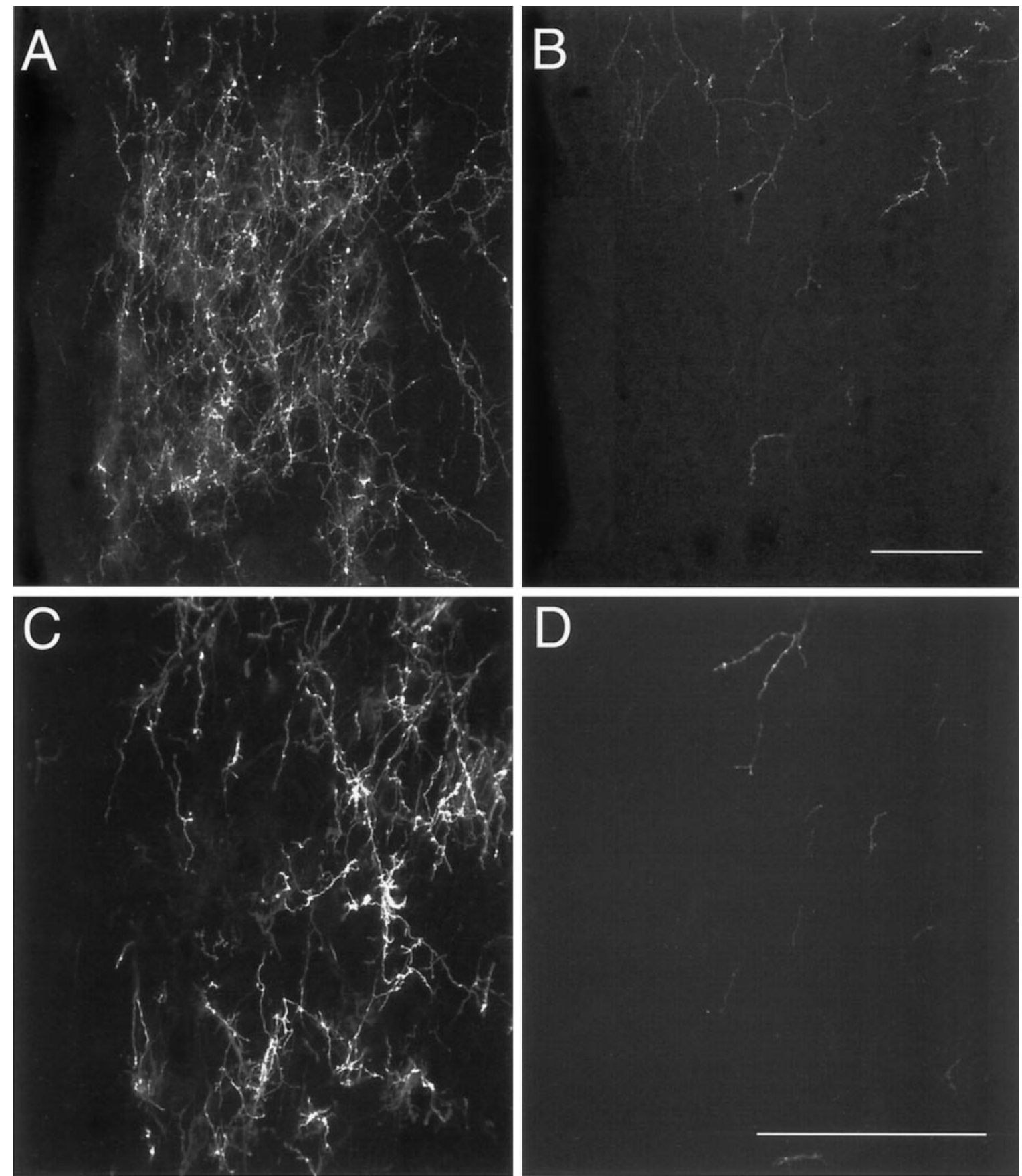

Figure 7. Confocal images of DiI-labeled fibers in the AVPV of P10 male $(A, C)$ and female $(B, D)$ rats. $A, B$, A maximum projection image was derived from 10 confocal images collected $(20 \times$ objective; NA, 0.75$)$ through a total distance of $20 \mu \mathrm{m}$. $C, D$, Ten confocal images were collected $(40 \times$ objective; NA, 1.3) through a total distance of $10 \mu \mathrm{m}$ to produce the projection image shown. Scale bars, $100 \mu \mathrm{m}$.

1971; Matsumoto, 1991). Clear exceptions to this general rule are some of the connections in the avian vocal control system (Konishi and Akutagawa, 1985) and the innervation of the dorsal tegmentum by laryngeal motor neurons in Xenopus frogs (Kelley, 1996), but differences in the mammalian brain tend to be more subtle. The projection from the BSTp to the AVPV is unique among hodological sex differences with respect to its magnitude, suggesting that it may be a particularly useful experimental model system for studying the development of sexually dimorphic connections in the mammalian forebrain.

Sex steroid hormones can influence the numbers of neurons in sexually dimorphic nuclei as well as the organization of their connections (Arnold and Jordan, 1988; Simerly, 1995). Thus, sexually dimorphic innervation of a target tissue may be controlled by regulating the survival of neurons that project to a target region. For example, in male zebra finches, neurons in the lateral magnocellular nucleus of the anterior neostriatum send a strong projection to the robust nucleus of the archistriatum. This projection is much weaker in females (Bottjer and Arnold, 1997), and axonal transport studies suggest that selective neuronal death of the neurons in the lateral magnocellular nucleus of the anterior neostriatum that provide inputs to the robust nucleus underlies 

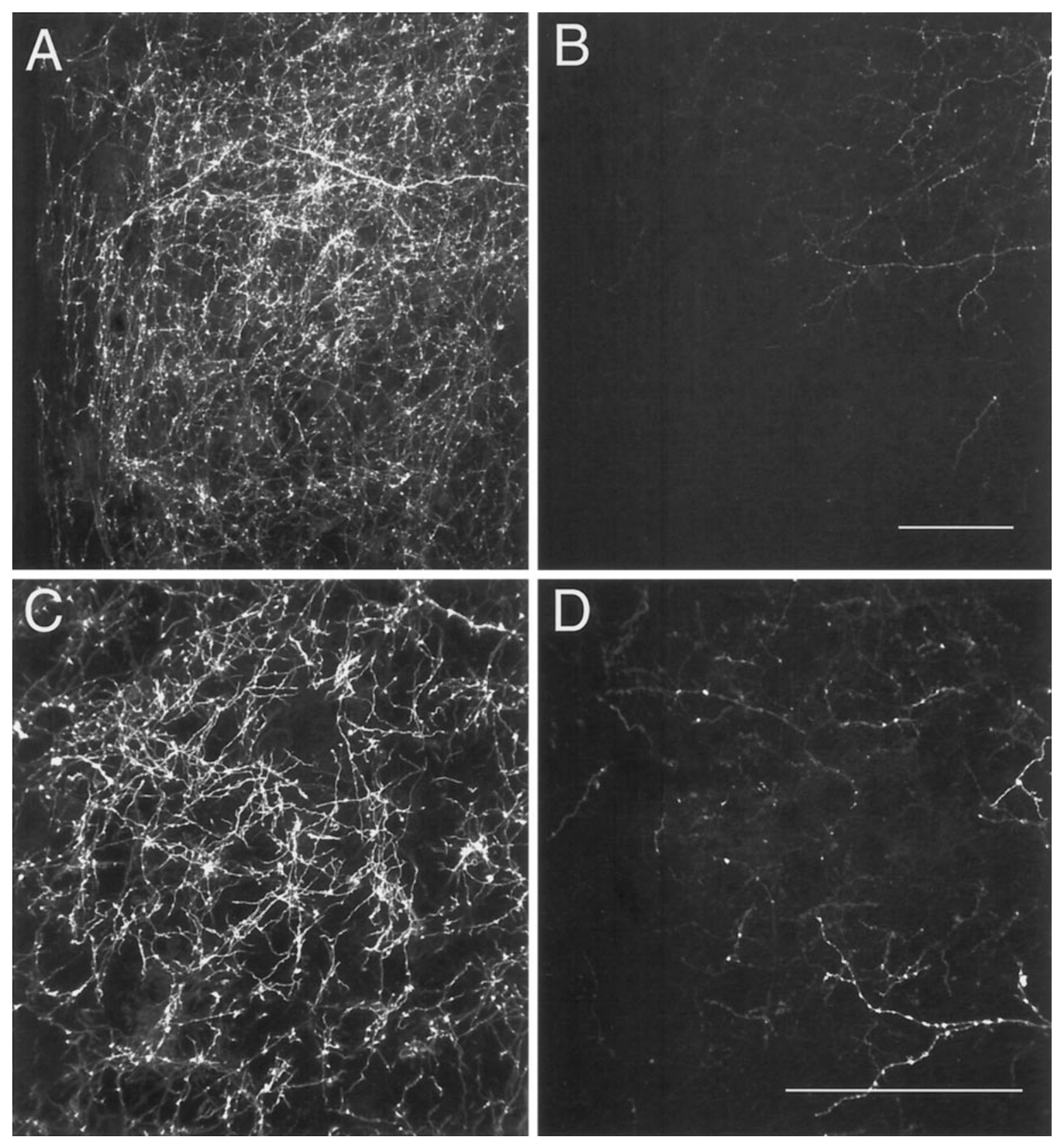

Figure 8. Confocal images of DiI-labeled fibers in the medial preoptic nucleus of P10 male $(A, C)$ and female $(B, D)$ rats. $A, B$, A maximum projection image was derived from six confocal images that were collected $(20 \times$ objective; NA, 0.75$)$ through a total distance of $12 \mu \mathrm{m}$. $C, D$, Ten images were collected (40× objective; NA, 1.3) through a total distance of $10 \mu \mathrm{m}$. Scale bars, $100 \mu \mathrm{m}$.

the development of this sexually dimorphic pathway (Nordeen et al., 1992). Similarly, testosterone is necessary for the survival of neurons in the spinal nucleus of the bulbocavernosus, which innervates the bulbocavernosus and levator ani muscles in the male rat. Nearly all of these neurons die in the female, but their survival and the maintenance of the innervation of the bulbocavernosus and levator ani muscles can be rescued by neonatal administration of testosterone (Breedlove, 1986). In both of these neural systems, development of a sexually dimorphic connection appears to be dependent on testosterone promoting the survival of a specific hormone-sensitive neuronal population. The BSTp of male rats is larger than that of females and appears to contain more neurons (del Abril et al., 1987), so it is not surprising to find stronger inputs to BSTp targets in the hypothalamus of male rats.
However, simply on the basis of the greater size of the BSTp, one would not predict the magnitude of the sexual dimorphism in the density of the projections to the AVPV. The BSTp also provides a stronger input to the MPNm in male rats than it does in females, but in contrast to its extremely weak projection to the AVPV, the BSTp sends a substantial input to the MPNm in females. Thus, the projection from the BSTp to the AVPV appears to be particularly dimorphic, relative to the other projections of the BSTp.

Numbers of projection neurons that survive into adulthood are often influenced by the size of their targets (Breedlove, 1985; Sohal, 1992; Thorn and Truman, 1994). Because the AVPV contains more neurons in females, it might be reasonable to expect that it would receive a stronger input from the BSTp in 


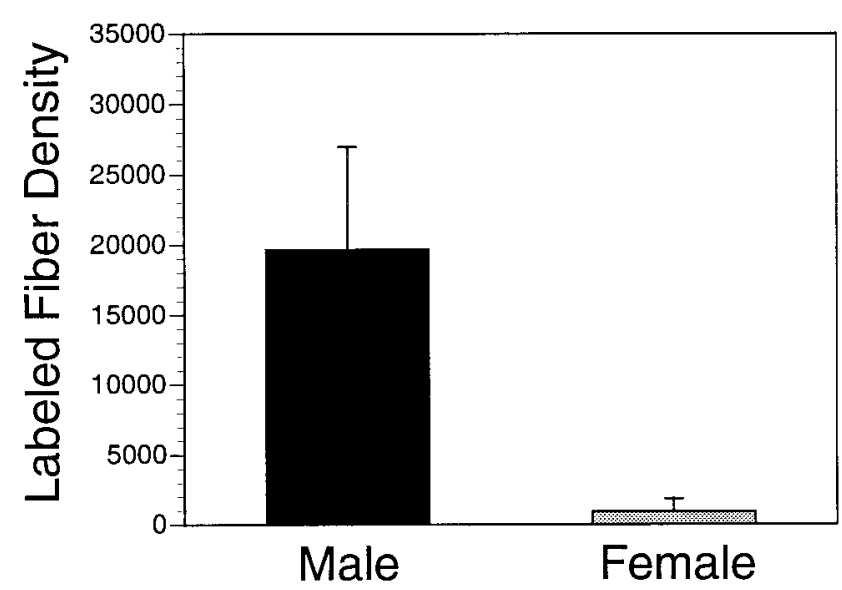

Figure 9. Graphical representation of the sexual dimorphism in the density of BSTp projections to the AVPV in P10 rats. The mean density of labeled fibers represents the mean of the total numbers of pixels in user-defined regions of thresholded, binarized images of DiI-labeled fibers, derived from a maximum projection image of 10 confocal image planes and collected with a $40 \times$ objective from three animals with comparable DiI implants centered in the BSTp.

animals of this sex because greater numbers of neurons in target regions tend to promote survival of neurons that provide afferent connections. However, the projection from the BSTp to the AVPV seems to be unique among sexually dimorphic pathways in that a smaller population of cells in the AVPV of males is innervated by a greater number of BSTp neurons, relative to the proportions of neurons that comprise this connection in females. Thus, the elevated levels of sex steroid hormones present in males during the perinatal period appear to decrease the number of neurons in the AVPV, while apparently promoting its innervation by neurons in the BSTp. This observation is consistent with the idea that sex steroid hormones can independently influence neuronal survival and the organization of connectivity within the same neural pathway. The result of the differential effect of sex steroids on the projection from the BSTp to the AVPV seems to be a dramatic convergence of sensory information on AVPV neurons in male rats.

The projection from the BSTp to the AVPV is established between postnatal days 9 and 10 in male rats, and both the density and distribution of labeled fibers are markedly different in females. By P10, the density of BSTp fibers innervating the AVPV is $\sim 20$-fold greater in males, whereas in females the few fibers that are present are clustered along the periphery of the nucleus. The projection from the BSTp to the MPNm develops a bit earlier than that to the AVPV. Labeled fibers were observed in the MPNm as early as P7 in male rats, but the adult pattern of innervation of the AVPV was not apparent until P10. The projections from the BSTp to the MPNm and the AVPV both appear to be relatively mature by $\mathrm{P} 10$.

For conceptual purposes the development of the projection from the BSTp to the AVPV can be divided into two phases. First, axons leave the BSTp and enter the medial preoptic area; this occurs in both sexes. Second, axons preferentially innervate the MPNm and AVPV in male rats but provide weaker inputs in females, suggesting that the specificity of the sexual differentiation lies in the second phase. Axons passing through the medial preoptic area appear to be attracted toward the MPNm and AVPV with very few axons deviating from their medial course. This type of directed axonal growth to target tissue is reminiscent of the development of thalamocortical projections to layer IV of the visual cortex (Agmon et al., 1993). Axons from the lateral geniculate nucleus enter layer IV of the visual cortex in bundles and terminate in specific target fields without sending collateral branches to inappropriate targets. In contrast, transient projections have been observed in corticospinal pathways (O'Leary, 1992). Although it remains possible that a transient connection between the BSTp and the AVPV may occur in the female, this seems to be unlikely because we did not observe substantial numbers of BSTp fibers in the AVPV of female rats at any of the ages examined. If such a transient connection occurs in females, it must be established and retracted between postnatal days 10 and 22. Our results suggest that very few axons from the BSTp ever reach the AVPV in females, and those that do are distributed along the lateral border of the nucleus, rather than being evenly dispersed within the AVPV as they are in the male. Similarly the BSTp may initially provide input to the AVPV and MPN in each sex, but the projection to the AVPV may be selectively pruned back during the perinatal period in females. This possibility also seems to be unlikely because the projections from the BSTp to the AVPV and MPNm seem to strengthen with postnatal age in both males and females, and a diffuse arbor of labeled axons is not observed in the MPNm or AVPV until the fibers reach their targets. Nor did axons projecting toward the MPNm and AVPV appear to be highly branched as they traversed the medial preoptic area. Thus, the development of the projections from the BSTp to the AVPV appears to be directed. Whether chemoattractive or chemorepulsive factors or contact with extracellular guidance molecules (Goodman, 1996) mediate the development of this sexually dimorphic pathway from the BSTp to the AVPV remains to be determined.

The function of the sexually dimorphic connection from the BSTp to the AVPV is not known, but presumably this pathway conveys olfactory information to the AVPV. Thus, the sexually dimorphic organization of afferent and efferent connections of the AVPV may contribute to sex-specific patterns of convergence or divergence of sensory information affecting neuroendocrine physiology. The exploration of molecular mechanisms underlying the development of this pathway represents a unique opportunity for gaining a greater understanding of how hormones regulate the architecture of the sexually dimorphic neural systems that regulate reproduction.

\section{REFERENCES}

Agmon A, Yang LT, O'Dowd DK, Jones EG (1993) Organized growth of thalamocortical axons from the deep tier of terminations into layer IV of developing mouse barrel cortex. J Neurosci 13:5365-5382.

Arai Y, Matsumoto A, Nishizuka M (1986) Synaptogenesis and neuronal plasticity to gonadal steroids: implications for the development of sexual dimorphism in the neuroendocrine brain. Curr Top Neuroendocrinol 7:291-307.

Arnold AP, Jordan CL (1988) Hormonal organization of neural circuits. In: Frontiers in neuroendocrinology, Vol 10 (Martini L, Ganong WF, eds), pp 185-214. New York: Raven.

Bottjer SW, Arnold AP (1997) Developmental plasticity in neural circuits for a learned behavior. Annu Rev Neurosci 20:459-481.

Breedlove SM (1985) Hormonal control of the anatomical specificity of motoneuron-to-muscle innervation in rats. Science 227:1357-1359.

Breedlove SM (1986) Cellular analyses of hormone influence on motoneuronal development and function. J Neurobiol 17:157-176.

del Abril A, Segovia S, Guillamón A (1987) The bed nucleus of the stria terminalis in the rat: regional sex differences controlled by gonadal steroids early after birth. Dev Brain Res 32:295-300.

Don Carlos LL, Handa RJ (1994) Developmental profile of estrogen receptor mRNA in the preoptic area of male and female neonatal rats. Dev Brain Res 79:283-289. 
Fink G (1988) Gonadotropin secretion and its control. In: The physiology of reproduction (Knobil E, Neill JD, eds), pp 1349-1376. New York: Raven.

Goodman CS (1996) Mechanisms and molecules that control growth cone guidance. Annu Rev Neurosci 19:341-377.

Gu GB, Simerly RB (1997a) Target specific hormonal regulation of sexually dimorphic projections from the principal nucleus of the bed nuclei of the stria terminalis. Soc Neurosci Abstr 23:341.

Gu GB, Simerly RB (1997b) Projections of the sexually dimorphic anteroventral periventricular nucleus in the female rat. J Comp Neurol 384:142-164.

Hsu SM, Raine L (1981) Protein A, avidin and biotin in immunohistochemistry. J Histochem Cytochem 29:1349-1353.

Hsu SM, Raine L, Fanger H (1981) The use of avidin-biotin-peroxidase complex $(\mathrm{ABC})$ in immunoperoxidase techniques: a comparison between $\mathrm{ABC}$ and unlabeled antibody (PAP) procedures. J Histochem Cytochem 29:577-580.

Ju G, Swanson LW (1989) Studies on the cellular architecture of the bed nuclei of the stria terminalis in the rat. I. Cytoarchitecture. J Comp Neurol 280:587-602.

Kelley D (1996) Sexual differentiation in Xenopus laevis. In: The biology of Xenopus (Tinsley RC, Kobel H, eds), pp 143-176. Oxford: Oxford UP.

Konishi M, Akutagawa E (1985) Neuronal growth, atrophy, and death in a sexually dimorphic song nucleus in the zebra finch brain. Nature 315:145-147.

Matsumoto A (1991) Synaptogenic action of sex steroids in developing and adult neuroendocrine brain. Psychoneuroendocrinology 16:25-40.

Matsumoto A, Arai Y (1986) Male-female difference in synaptic organization of the ventromedial nucleus of the hypothalamus in the rat. Neuroendocrinology 42:232-236.

McAbee M, Don Carlos LL (1996) Hormonal regulation of androgen receptor (AR) mRNA in the neonatal rat forebrain. Soc Neurosci Abstr 22:558.

Neill JD, Nagy GM (1994) Prolactin secretion and its control. In: The physiology of reproduction (Knobil E, Neill JD, eds), pp 1833-1860. New York: Raven.

Nordeen EJ, Grace A, Burek MJ, Nordeen KW (1992) Sex-dependent loss of projection neurons involved in avian song learning. J Neurobiol 23:671-679.
O’Leary DDM (1992) Development of connectional diversity and specificity in the mammalian brain by the pruning of collateral projections. Curr Opin Neurobiol 2:70-77.

Raisman G, Field PM (1971) Sexual dimorphism in the preoptic area of the rat. Science 173:731-733.

Segovia S, Guillamón A (1993) Sexual dimorphism in the vomeronasal pathway and sex differences in reproductive behaviors. Brain Res Rev 18:51-74.

Shinoda K, Nagano M, Osawa Y (1994) Neuronal aromatase expression in preoptic, strial, and amygdaloid regions during late prenatal and early postnatal development in the rat. J Comp Neurol 343:113-129.

Simerly RB (1990) Hormonal control of neuropeptide gene expression in sexually dimorphic olfactory pathways. Trends Neurosci 13:104-110.

Simerly RB (1995) Hormonal regulation of limbic and hypothalamic pathways. In: Neurobiological effects of sex steroid hormones (Micevych PE, Hammer Jr RP, eds), pp 85-114. Cambridge, UK: Cambridge UP.

Simerly RB (1998) Organization and regulation of sexually dimorphic neuroendocrine pathways. Behav Brain Res, in press.

Simerly RB, Swanson LW (1988) Projections of the medial preoptic nucleus: a Phaseolus vulgaris leucoagglutinin anterograde tract-tracing study in the rat. J Comp Neurol 270:209-242.

Simerly RB, Chang C, Muramatsu M, Swanson LW (1990) Distribution of androgen and estrogen receptor mRNA-containing cells in the rat brain: an in situ hybridization study. J Comp Neurol 294:76-95.

Sohal GS (1992) The role of target size in neuronal survival. J Neurobiol 23:1124-1130.

Sumida H, Nishizuka M, Kano Y, Arai Y (1993) Sex differences in the anteroventral periventricular nucleus of the preoptic area and in the related effects of androgen in prenatal rats. Neurosci Lett 151:41-44.

Swanson LW (1992) Brain maps: structure of the rat brain. Amsterdam: Elsevier Science.

Thorn RS, Truman JW (1994) Sexual differentiation in the CNS of the moth, Manduca sexta. II. Target dependence for the survival of the imaginal midline neurons. J Neurobiol 25:1054-1066.

Wiegand SJ, Terasawa E (1982) Discrete lesions reveal functional heterogeneity of suprachiasmatic structures in regulation of gonadotropin secretion in the female rat. Neuroendocrinology 34:395-404. 